\title{
Understanding the magnetic anisotropy in Fe-Si amorphous alloys
}

\author{
J. DIAZ, N.M. HAMDAN, P. JALIL, Z. HUSSAIN, S. M. VALVIDARES and J. M. ALAMEDA
}

\begin{abstract}
The origin of the magnetic anisotropy in a very disordered Fe-Si alloy has been investigated. The alloy containing $40 \%$ at. Si was prepared in the form of a thin film in a DC magnetron sputtering chamber. Structural disorder was obtained from Extended X-ray Absorption Fine Structure spectroscopy. The uniformity and lack of inhomogeneities at a microscopic level was checked by measuring their transverse magnetic susceptibility and hysteresis loops. The orbital component of the magnetic moment was measured by X-ray Magnetic Circular Dichroism spectroscopy. The orbital moment was extraordinary high, $0.4 \mu_{\mathrm{B}}$. Such a high value contrasted with the relatively small uniaxial anisotropy energy of the thin film $\left(2 \mathrm{~kJ} / \mathrm{m}^{3}\right)$. This suggests that the cause of the magnetic anisotropy in this alloy was a small degree of correlation in the orientation of the local orbital moments along a preferential direction.
\end{abstract}

Index Terms-Magnetic films, amorphous materials, magnetic anisotropy, $x$-ray spectroscopies.

\section{INTRODUCTION}

The Random Anisotropy model is the starting point to understand the magnetic properties of amorphous elements and compounds [1,2]. In essence, the magnetic properties of these materials are the result of the competition between the ferromagnetic exchange interaction and the uniaxial magnetocrystalline anisotropy of each site. The magneto-crystalline anisotropy will try to pin the spin moment to the direction of anisotropy of each particular site whereas the exchange coupling will try to align the spins from site to site. As a consequence, the spins will be spatially correlated (aligned) over a characteristic length, that will be longer the stronger is the exchange interaction compared to the magneto-crystalline anisotropy. Rare Earth (RE)-Transition Metal (TM) compounds are the most used materials to test this model because of the high local magnetic anisotropy of the RE [3]. The TM is introduced to increase the exchange interaction. Then, the ferromagnetic correlation lengths can be controlled by changing the relative concentrations of the

Manuscript received February 14, 2002. This work was supported in part by the Spanish CICYT under Grant MAT99-0724 and HF/1999-041.

J. Díaz, S.M. Valvidares and J. M. Alameda are with Dpto. Física, Universidad de Oviedo, 33007 Oviedo, SPAIN. E-mail: jdiaz@condmat01.geol.uniovi.es).

N. M. Hamdan, P. Jalil, and Zahid Hussain are with Advanced Light Source Synchrotron, Lawrence Berkeley National Laboratory, 1, Cyclotron Road, Berkeley, CA- 94720 , USA transition metal. $3 \mathrm{~d}$ electrons in transition metals have a much weaker spin-orbit interaction compared to the Rare Earths, and they interact more strongly with the crystal field which makes their orbital moments smaller so that their magnetism is dominated by the exchange interaction. In Transition MetalMetalloid (TM-M) alloys, the Metalloid ( $\mathrm{Si}, \mathrm{B}, \mathrm{P}$. .) is introduced to amorphisize the TM structure. The exchange interaction is believed to still dominate in these compounds [2]. Their ferromagnetic correlation length is longer than in the RE-TM alloys, which also implies that their spatial spinspin correlation function decays more slowly. These materials have a quasi long range magnetic order and, also due to the dominant exchange interaction, they behave as very soft ferromagnets, i.e., their coercivity and anisotropy energy are very small. The TM-M alloys prepared in the form of thin films usually have a well defined easy axis that can not come about from the pure exchange interaction which is isotropic. The anisotropy of the films should come from either the spinorbit interaction, responsible of the magneto-crystalline anisotropy at each site, or from magnetic dipolar interactions [2]. The last one can be originated at an atomic level due to structural anisotropies, or on a microscopic scale, due to defects and inhomogeneities. Identifying the factors that cause the anisotropy in these films is important from a practical point of view because it allows the control of their magnetic characteristics.

The perpendicular anisotropy observed in amorphous $\mathrm{TbFe}_{2}$ compounds is caused by structural anisotropy, namely the pairing of $\mathrm{Fe}$ and $\mathrm{Tb}$ neighbor atoms along well defined directions [4]. On the other hand, the spin-orbit interaction is the origin of the anisotropy observed in many TM compounds. Large orbital moments have been measured precisely in compounds with low symmetry environments, as in $\mathrm{YCo}_{5}$ [5,6] or $\mathrm{Au} / \mathrm{Co} / \mathrm{Au}$ multilayers [7], and they were found to be the cause of their strong magnetic anisotropies. The environments of amorphous alloys should be of low symmetry so that large values of their orbital moments would be expected. The experimental determination of the orbital component of the magnetic moment is a difficult measurement, specially in non-crystalline solids and thin films. That might be the reason why there is little experimental information about the magnitude of the orbital moments in TM based amorphous alloys until recently. This has been largely due to the development of the X-ray Magnetic Circular Dichroism (XMCD) technique, which allows the direct measurement of the orbital component of the magnetic moment by applying rather simply sum rules to the spectra taken at the $L$ edge of the TM element with left and 
right circular polarization of the incident $\mathrm{x}$-rays $[8,9]$. The high sensitivity of this technique permits measurement of the magnetic moments even in ultra-thin films [7]. Measurements obtained with this method in amorphous Fe-Ni-Si-B ribbons showed orbital moments for $\mathrm{Fe}$ as high as $0.17 \mu_{\mathrm{B}}$ (Bohr magnetons) [10]. This paper presents XMCD measurements in an $\mathrm{Fe}_{60} \mathrm{Si}_{40}$ amorphous thin film that yield a value for the orbital moment of $\mathrm{Fe}$ that was even higher, comparable to that of $\mathrm{Co}$ in $\mathrm{YCo}_{5}$. The possible effect of this large orbital moment in the magnetic properties of the film and the origin of its magnetic anisotropy is qualitatively discussed in terms of its EXAFS spectrum, transverse magnetic susceptibility and hysteresis loops.

\section{EXPERIMENTAL}

The sample was obtained by evaporating $\mathrm{Fe}$ and $\mathrm{Si}$ from separate DC magnetron sputtering guns on a polished fused quartz substrate. The thin film was prepared at room temperature under an Ar pressure of $10^{-3} \mathrm{mbar}$, with a system base pressure of $10^{-8}$ mbar. The distance between the magnetron guns and the sample was of $200 \mathrm{~mm}$, the deposition rate was of $0.1 \mathrm{~nm} / \mathrm{s}$, and the thickness of the film was 200 $\mathrm{nm}$. This film was the one with the highest structural disorder from a set of samples prepared at different $\mathrm{Fe}$ and $\mathrm{Si}$ concentrations, as deduced from their Extended X-ray Absorption Fine Structure (EXAFS) spectra [11]. The film exhibited the largest dispersion in the distance between first neighbors with no order to the second neighbors. The sample had a well defined uniaxial magnetic anisotropy with the easy axis in the plane of the sample. X-ray Photoemission Spectroscopy (XPS) and X-ray Magnetic Circular Dichroism (XMCD) analysis of the sample was performed on beam line 9.3.2 at the Advanced Light Source Synchrotron [12]. Right and left circular polarization was obtained by selecting the part of the synchrotron radiation emitted from a bending magnet above or below the electron orbit plane, moving vertically a slit set at the front end of the beam line. The degree of circular polarization was $80 \%$. XPS spectra were obtained with linear polarized light. X-ray absorption spectra (XAS) were obtained by measuring the photocurrent emitted from the sample. The XMCD signal at the Fe $L$ edge was obtained by subtracting the normalized XAS spectra taken at different circular polarizations (Left-Right). The XMCD signals measured from the spectra taken at 0 and $\pi$ azimuthal orientations of the sample and at a fixed circular polarization (either Left or Right) were all equivalent. The spectra were taken with the sample at magnetic remanence. The ratio between the magnetic moment of the film in remanence with respect to its saturation magnetic moment was 0.96 . The sample was exposed to air before introducing it in the analysis chamber. The relative concentrations of Fe and $\mathrm{Si}$ in the film were obtained by X-ray Photoemission Spectroscopy. A larger than expected concentration of Si $(85 \%)$ was found at the surface of the thin film. This $\mathrm{Si}$ rich surface layer was removed by sputtering the film with $\mathrm{Ar}$ ions of $1 \mathrm{keV}$ for 15 min. with an ion current of $5 \mu \mathrm{A}$. The concentration of Si after Ar sputtering was $40 \%$ and no traces of oxygen or carbon were found. Sputtering was performed at remanence.
Observations made by XMCD indicated a loss of about $20 \%$ in the magnitude of the magnetic moment after sputtering. The XAS spectra presented here were taken after re-magnetizing the sample after Ar sputtering. The angle of incidence of the $\mathrm{x}$-rays was $70^{\circ}$ with respect to the sample normal. The transverse magnetic susceptibility and hysteresis loops of the sample were measured in air using the transverse magnetooptical Kerr effect [13].

\section{RESULTS AND DISCUSSION}

Figure 1 shows the XAS Fe $L$ edge spectra of the sample taken at Left and Right circular polarization along the easy axis and the resulting XMCD signal, after correction of incidence angle and degree of circular polarization. The orbital $\left(\mathrm{m}_{\text {orb }}\right)$ to spin $\left(\mathrm{m}_{\text {spin }}\right)$ magnetic moment ratio was obtained from applying the sum rules:

$$
\begin{gathered}
\mathrm{m}_{\text {orb }}=-2 \mu_{B}(\mathrm{~A}+\mathrm{B}) / \mathrm{C} \\
\mathrm{m}_{\text {spin }}=-3 \mu_{B}(\mathrm{~A}-2 \mathrm{~B}) / \mathrm{C}
\end{gathered}
$$

were $\mathrm{A}$ and $\mathrm{B}$ are the areas of the XMCD peaks, shown in figure 1 , and $\mathrm{C}$ is a constant. The orbit to spin moment ratio was 0.25 , almost a factor of 1 larger than that observed in bcc Fe. Absolute values of the spin and orbital moments were estimated using the XMCD spectra of bcc Fe published by Chen et al. [8]. This gave a value for the spin component of $1.6 \mu_{B}\left(2 \mu_{B}\right.$ in bcc $\left.\mathrm{Fe}\right)$ and a huge orbital moment of $0.4 \mu_{B}$, almost five times larger than the orbital moment in bcc Fe $\left(0.085 \mu_{B}\right)$. This orbital moment is comparable to that measured for $\mathrm{Co}$ in $\mathrm{YCo}_{5}$ crystals [6], or the extrapolated value for $\mathrm{Co}$ if one monolayer of $\mathrm{Co}$ was sandwiched in $\mathrm{Au}$ $(\mathrm{Au} / \mathrm{Co} / \mathrm{Au})[7]$. The strongly anisotropic local environment of Fe ions, caused by the structural disorder, is likely to be the origin of such a large orbital moment. The local anisotropy is directly linked to the magnitude of the orbital moment. A rough estimation of the local anisotropy constant will yield a value of the order of $K_{l o c}=10^{6} \mathrm{~J} / \mathrm{m}^{3}$, taking as a reference the anisotropy constant of $\mathrm{Fe}_{3} \mathrm{Si}\left(K=4 \cdot 10^{5} \mathrm{~J} / \mathrm{m}^{3}\right)$. Previous experiments in Fe-Si [13] and Y-Co [14] amorphous alloys suggested that their local anisotropy constants should be of the order of $10^{6} \mathrm{~J} / \mathrm{m}^{3}$. This value of the local anisotropy, being large, is still two orders of magnitude lower than the local anisotropy for Rare Earth-Iron alloys. Since the exchange stiffness parameter is of the same order in both types of alloys $\left(A_{e x}=10^{-11} \mathrm{~J} / \mathrm{m}\right)$, this Fe-Si amorphous alloy should be still in the limit of quasi long range ferromagnetic order. The ferromagnetic correlation length $l_{f}$ for this alloy was about $13 \mu \mathrm{m}$, using the relation obtained from the random anisotropy model $[1,2]$ :

$$
l_{f}=16 A_{e x}{ }^{2} / 9 K_{l o c}{ }^{2} d^{3}
$$

where $d$ is the structural correlation length, which was considered of the order of interatomic distances. This ferromagnetic correlation length is much larger than that found in Rare-Earth-Transition Metal amorphous alloys such as $\mathrm{TbFe}_{2}\left(l_{f}=50 \mathrm{~nm}\right)[3]$.

The transverse susceptibility measurements showed that the sample was uniaxial with little dispersion in the easy axis. The anisotropy field $\mathrm{H}_{\mathrm{k}}$, deduced from linear interpolation of the inverse susceptibility function, was $1.93 \mathrm{kA} / \mathrm{m}$ if the DC field $\mathrm{H}_{\mathrm{dc}}$ was applied along the hard axis, and $1.81 \mathrm{kA} / \mathrm{m}$ when 
applied along the easy axis (see figure 2). The difference between the two anisotropy fields is due to the ripple induced by the dispersion in the easy axis. This "ripple field" was only $55.7 \mathrm{~A} / \mathrm{m}$, i.e., $3 \%$ of the anisotropy field. The hysteresis loop obtained parallel to the easy axis showed a remanence ratio of 0.96 (see figure 3(a)), consistent with a well defined easy axis. The coercive field was relatively small, only $51.7 \mathrm{~A} / \mathrm{m}$. The hysteresis loop along the hard axis (figure 3(b)) differs strongly from that expected in a perfect uniaxial thin film, i.e., a straight line connecting $-\mathrm{M}_{\mathrm{s}}$ to $\mathrm{M}_{\mathrm{s}}$ from $-\mathrm{H}_{\mathrm{k}}$ to $\mathrm{H}_{\mathrm{k}}$. The strong curvature of the loop does not fit well to laws of the type $1 / \mathrm{H}$ or $1 / \mathrm{H}^{2}$, associated with the magnetization behavior of nanocrystalline ferromagnets with inhomogeneities in it [15]. The hysteresis loop resembles the behaviour of a paramagnet, with the exception of the coercive part at low fields $(<95.5 \mathrm{~A} / \mathrm{m})$. Actually, the hysteresis loop is well fitted by an exponential function. This curvature should reflect the large disorder in the local moments at an atomic level. These measurements suggest that the sample is very uniform with no inhomogenities and with disorder on a very short scale.

The anisotropy energy $K_{u}$, associated with the easy axis, was deduced from the relation:

$$
K_{u}=\mathrm{H}_{\mathrm{k}} \mathrm{M}_{\mathrm{s}} / 2
$$

where $M_{s}$ is the magnetic moment density of the sample at the saturation field. This was about $1.25 \mathrm{~Wb} / \mathrm{m}^{2}$, using the magnetic moment for $\mathrm{Fe}$ ions deduced from the XMCD spectra $\left(2 \mu_{B}\right) . K_{u}$ was about $2 \mathrm{~kJ} / \mathrm{m}^{3}$, of the same order of magnitude than of CoTi amorphous alloy, which is among the most anisotropic TM amorphous alloys. However, it is very small compared to the anisotropy of bcc $\mathrm{Fe}\left(50 \mathrm{~kJ} / \mathrm{m}^{3}\right)$ and much lower than that in $\mathrm{YCo}_{5}\left(10^{3} \mathrm{~kJ} / \mathrm{m}^{3}\right)$. Why, having such a large local anisotropy, is the uniaxial anisotropy of this $\mathrm{Fe}-\mathrm{Si}$ amorphous alloy so small, relatively? The answer should be that the random orientation of the axis at each site, together with the large exchange interaction, notably reduces the macroscopic or effective anisotropy with respect to the local anisotropy. However, certain orientational correlation in one preferential direction between distant sites might exist, and this could be the cause of the observed uniaxial anisotropy.

A relatively small degree of orientational correlation could be enough to produce the uniaxial anisotropy of the film, given the high value of the local anisotropy energy $K_{l o c}$ with respect to the effective anisotropy energy $K_{u}$. Actually, computer simulations show that such an arrangement of local axes is energetically less favorable than other arrangements where there exist a certain orientational correlation, i.e., the random anisotropy is not averaged away [1]. Orientational correlation lengths can be longer than spatial correlation lengths, as has been experimentally observed [16]. Other mechanism that could induce an effective magnetic anisotropy in the $\mathrm{Fe}-\mathrm{Si}$ amorphous alloys could be strain or unhomogeneities. But they were discarded in this analysis because the energy and direction of the uniaxial anisotropy of the films did not depend on the substrate or the azimutal orientation of crystalline substrates.

\section{Conclusions}

In summary, XMCD measurements showed extraordinary large values for the orbital momentum in $\mathrm{Fe}-\mathrm{Si}$ amorphous alloys. The relatively small anisotropy energy of these films indicates that the local moments are largely disordered. The origin of their uniaxial anisotropy should be a certain degree of correlation in the orientation of the local moments along a preferential direction.

\section{REFERENCES}

[1] R. Alben et al. J. Appl. Phys. 49, 1653 (1978)

[2] T. Kaneyoshi. "Introduction to Amorphous Magnets" World Scientific Publishing, Singapore (1992)

[3] F. Hellman et al. Phys. Rev. B 59, 11408 (1999)

[4] V. G. Harris et al. Phys. Rev. Lett. 69, $1939(1992)$

[5] J. M. Alameda et al. J. Magn. Magn. Mat. 15-18, 1257 (1980)

[6] J. Schweizer et al. J. Phys. F 10, 2799 (1980)

[7] D. Weller et al. Phys. Rev. Lett. 75, 3752 (1995)

[8] C. T. Chen et al. Phys. Rev. Lett. 75,152 (1995)

[9] J. Stöhr and H. König. Phys. Rev. Lett. 75, 3748 (1995)

[10] M. Knobel et al. J. Mag. Mag. Mat. 233,78 (2001)

[11] J. Díaz et al. J. Mag. Mag. Mat. (JEMS 2001 Conference) (To be published)

[12] Z. Hussain et al. J. Electron Spect. and Rel. Phen. 80,401 (1996)

[13] J. M. Alameda et al. Phys. Stat. Sol. (a) 85, 511 (1984)

[14] J. M. Alameda et al. J. Mag. Mag. Mat. 67,115 (1987)

[15] G. R. Aranda et al. J. Appl. Phys. 83, 6341 (1998)

[16] E. M. Chudnovsky and J. Tejada. Europhys. Lett. 23, 517 (1993)

\section{Figure Captions}

Fig. 1. X-ray absorption spectra at the Fe $L$ edge obtained with left $\left(\mu^{+}\right)$and right $\left(\mu^{-}\right)$circular polarized light of an amorphous $\mathrm{Fe}_{60} \mathrm{Si}_{40}$ thin film, their substraction, i.e., the XMCD spectrum, and the integration of the XMCD spectrum.

Fig. 2. Inverse Transverse Susceptibility vs. field: solid dots, field applied along the hard axis $\left(\chi^{-1}(0)\right)$; hollow dots, field applied along the easy axis $\left(\chi^{-1}(\pi / 2)\right)$.

Fig. 3. Hysteresis loops of a 200 nm thick $\mathrm{Fe}_{60} \mathrm{Si}_{40}$ amorphous film along (a) the easy axis, (b) the hard axis. Note the different Magnetic Field scale for each Hysteresis loop. 
349 (USERNAME: JDIAZ)

4

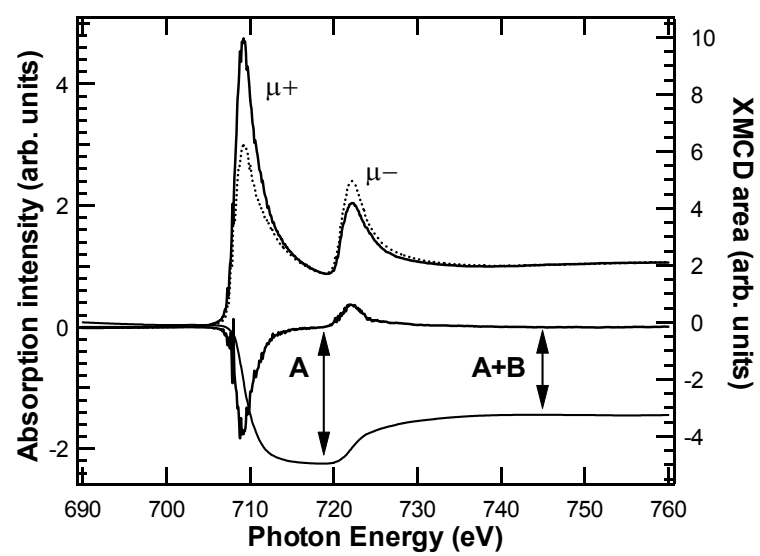

Fig. 1. X-ray absorption spectra at the $\mathrm{Fe} L$ edge with left $(\mu+)$ and rit $(\mu-)$ circular polarized light of an amorphous $\mathrm{Fe}_{60} \mathrm{Si}_{40}$ thin film, their subtraction, ie., the XMCD spectrum, and the integration of the XMCD spectrum.

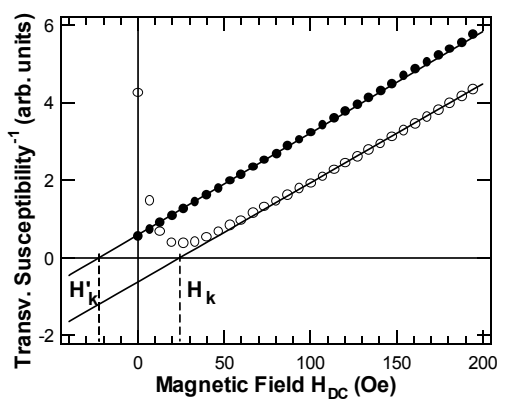

Fig. 2. Inverse transverse susceptibility vs field: solid dots, field applied along the hard axis $\left(\chi_{t}^{-1}(0)\right)$, hollow dots, field applied along the easy axis $\left(\chi_{t}^{-1}(\pi)\right)$.

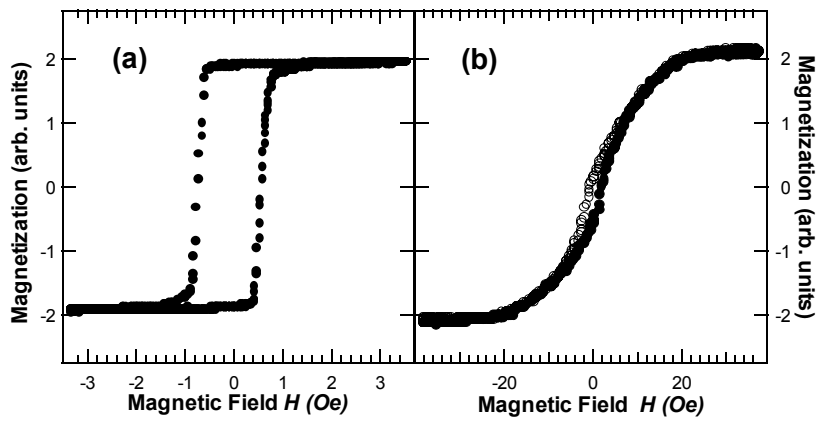

Fig. 3. Hysteresis loops of a $200 \mathrm{~nm}$ thick $\mathrm{Fe}_{60} \mathrm{Si}_{40}$ amorphous film along (a) the easy axis, (b) the hard axis. Note the different Magnetic Field scale for each hysteresis loop. 